Sharif University of Technology
Scientia Iranica
SCIENTIA $\quad \begin{gathered}\text { Transactions D: Computer Science \& Engineering and Electrical Engineering } \\ \text { w }\end{gathered}$

\title{
Viable arcuate double-sided magnetic gear for competitive torque density transmission capability
}

\author{
S.A. Afsari, H. Heydari* and E. Bashar \\ Center of Excellence for Power System Automation and Operation, Department of Electrical Engineering, Iran University of Science \\ and Technology (IUST), Tehran, P.O. Box 16846-13114, Iran.
}

Received 27 July 2014; accepted 25 August 2015

\section{KEYWORDS \\ Axial flux; \\ Arcuate; \\ Cogging torque; \\ Magnetic gear; \\ Double-sided; \\ Torque density.}

\begin{abstract}
Magnetic Gears (MGs) offer significant potential advantages relative to conventional mechanical gears, such as: no contact, no friction, no lubrication, inherent overload protection, and freeness from noise and vibration. Despite these superior features, they have received little attention due to complexity of operation and relatively low torque density transmission. This paper introduces a new magnetic gear topology with a highly competitive torque density transmission capability. This configuration is inspired by combination of the traditional radial and axial flux MGs. The assurance of accuracy based on a basic design of axial flux magnetic gear dimensions leads to Proof-of-Concept "Arcuate Double-Sided MG". The Proof-of-Concept seeks to provide a better solution to the problems of flux maldistribution and concentration compared to what has been proposed previously. Thus, torque density performance of this MG is intensively augmented. The cogging torque is also computed in further simulations. However, this requires advanced numerical techniques and, hence, 3-dimensional simulations for calculating localized flux density and the corresponding torque density. Completion of this development will mark an important milestone in magnetic gear technology, and significant performance improvement will be realized.
\end{abstract}

(C) 2016 Sharif University of Technology. All rights reserved.

\section{Introduction}

The main purpose of any gearing system is to convert between torque and speed. One of the leading and most important features of Magnetic Gears (MGs) is the fact that the produced torque on the driven member exceeds that of the driver. These gears can be used in many systems, such as automobiles, to convert the high speed of rotary crank shaft in the inner combustion engine to a relatively high torque at the wheels and/or in wind turbines to convert the high torque and slow rotational speed of turbine blades into the high speed required by typical generators.

Magnetic gearing boasts a plethora of advan-

*. Corresponding author. Tel.: +9821 77208123;

E-mail address: heydari@iust.ac.ir (H.Heydari) tages over standard mechanical gearing, such as bidirectional and contactless power transfer, oil-free operation, inherent overload protection, potential for high efficiency, and little or no maintenance. Moreover, input and output shafts can be isolated, offering additional option to the mechanical designers. They further support a wide range of gear ratios and integration with electrical generators and motors [1-3].

In spite of the aforementioned advantages, MGs still suffer from chronic limitations, such as complexity and poor torque density $[4,5]$.

In order to assess the novelty of this paper, a thorough knowledge of the previous existing mainbased publications is well thought-out, upon which the proposed approach improves or from which it diverges.

Atallah et al. [6,7] report a radial flux magnetic gear with coaxial structure and then axial flux 
magnetic gear. Both types have three main parts: an external (low-speed) rotor made of higher number of permanent magnets, an inner (high-speed) rotor made of lower number of permanent magnets, and a modulator, which consists of several soft magnetic pole pieces for modulating magnetic flux distribution between the rotors.

In most applications, such as hybrid electric vehicles and wind turbines, due to space limitation, having a magnetic gear with higher torque density is inevitable. Many studies have been done to improve performances of magnetic gears. For instance, in a relevant literature, Jian et al. [8] proposed an optimum design method for improving the modulating effect of Coaxial Magnetic Gear (CMG) aiming at delivering the full mechanical torque. They used surface response methodology to model the relationship between the maximum pull-out torque and shape factors leading to an optimum shape of the ferromagnetic segments.

Other related strands of prior arts are on the optimization to model optimized magnetic gears. Fukuoka et al. [9] studied a method for design optimization of the surface-mounted permanent magnet type magnetic gear using reluctance network analysis, aiming at an optimal design for maximum torque. Jian et al. [10] in another study used High Temperature Superconducting (HTS) technology by designing a bulk HTS modulator. The bulk HTS offered not only the desired field modulation effect and higher transmitted torque, but also suppressed the undesirable end-effects. In another investigation [11], they proposed a coaxial magnetic gear with Halbach PM Arrays offering at least $13 \%$ higher torque density, up to $67 \%$ lower cogging torque, and typically $28 \%$ lower iron losses than those of a conventional one.

This paper will push the state-of-the-art by offering an innovative approach of MG and inspiration of combination of the axial and radial flux topologies for enhancing its torque density.

Moreover, in this study, a double-sided axial flux MG regarded as a Proof-of-Concept is thoroughly analyzed. The cogging torque is computed in further simulations. Hence comes the need for advanced numerical techniques and simulation studies in a viable Finite Element Method (FEM) analysis in an exemplary basic design of axial flux MG, which is well thought-out as a precious guide. This is followed by a novel design topology, called "Arcuate Double-Sided MG" (ADSMG), of the same size leading to much higher torque density and efficiency.

\section{Principle operation of magnetic gear}

The operating principle of the proposed approach bears resemblance to the rotary and linear MGs [12]. In an MG, the magnetic fields are produced by the high- or low-speed permanent magnet rotors, modulated via ferromagnetic pole pieces such that the appropriate space harmonics have the requisite number of poles, acting with the other permanent magnet rotor.

The mathematical formulation of the radial flux density at angle $\theta$ produced by each permanent magnet rotor of an axial flux MG, without modulation function, can be written as [13]:

$$
B_{r}^{\prime}(r, \theta)=\sum_{m=1,3,5, \cdots} b_{r m}(r) \cos \left(m p\left(\theta-\Omega_{r} t\right)+m p \theta_{0}\right)
$$

The radial component of modulation function can be written as:

$$
\lambda_{r}(r, \theta)=\lambda_{r 0}(r)+\sum_{j=1,2,3, \cdots} \lambda_{r j}(r) \cos \left(j n_{s}\left(\theta-\Omega_{s} t\right)\right) .
$$

Finally, the radial component of flux distribution can be given by:

$$
\begin{aligned}
& B_{r}(r, \theta)=B_{r}^{\prime}(r, \theta) \times \lambda_{r}(r, \theta) \\
& =\lambda_{r 0} \sum_{m=1,3,5, \cdots} b_{r m}(r) \cos \left(m p\left(\theta-\Omega_{r} t\right)+m p \theta_{0}\right) \\
& +\frac{1}{2} \sum_{m=1,3,5, \cdots j=1,2,3, \cdots} \lambda_{r j}(r) b_{r m} \cos \left(\left(m p+j n_{s}\right)\right. \\
& \left.\quad \times\left(\theta-\frac{m p \Omega_{r}+j n_{s} \Omega_{s}}{m p+j n_{s}} t\right)+m p \theta_{0}\right) \\
& +\frac{1}{2} \sum_{m=1,3,5, \cdots j=1,2,3 \cdots} \lambda_{r j}(r) b_{r m} \cos \left(\left(m p-j n_{s}\right)\right. \\
& \left.\quad \times\left(\theta-\frac{m p \Omega_{r}-j n_{s} \Omega_{s}}{m p-j n_{s}} t\right)+m p \theta_{0}\right) .
\end{aligned}
$$

Similarly, the circumferential flux density distribution and modulation function can be written, respectively, as:

$$
\begin{aligned}
& B_{\theta}^{\prime}(r, \theta)=\sum_{m=1,3,5, \cdots} b_{\theta m}(r) \sin \left(m p\left(\theta-\Omega_{r} t\right)+m p \theta_{0}\right) \\
& \lambda_{\theta}(r, \theta)=\lambda_{\theta 0}(r)+\sum_{j=1,2,3, \cdots} \lambda_{\theta j}(r) \cos \left(j n_{s}\left(\theta-\Omega_{s} t\right)\right) .
\end{aligned}
$$

As a result, the circumferential component of flux distribution is given by:

$$
\begin{aligned}
& B_{\theta}(r, \theta)=B_{\theta}^{\prime}(r, \theta) \times \lambda_{\theta}(r, \theta) \\
& =\lambda_{\theta 0} \sum_{m=1,3,5, \cdots} b_{\theta m}(r) \sin \left(m p\left(\theta-\Omega_{r} t\right)+m p \theta_{0}\right)
\end{aligned}
$$




$$
\begin{aligned}
& +\frac{1}{2} \sum_{m=1,3,5, \cdots} \sum_{j=1,2,3 \cdots} \lambda_{\theta j}(r) b_{\theta m} \sin \left(\left(m p+j n_{s}\right)\right. \\
& \left.\times\left(\theta-\frac{m p \Omega_{r}+j n_{s} \Omega_{s}}{m p+j n_{s}} t\right)+m p \theta_{0}\right) \\
& +\frac{1}{2} \sum_{m=1,3,5, \cdots j=1,2,3 \cdots} \lambda_{\theta j}(r) b_{\theta m} \sin \left(\left(m p-j n_{s}\right)\right. \\
& \left.\times\left(\theta-\frac{m p \Omega_{r}-j n_{s} \Omega_{s}}{m p-j n_{s}} t\right)+m p \theta_{0}\right)
\end{aligned}
$$

where, $p$ is the number of pole pairs of the permanent magnet rotor, $n_{s}$ is the number of modulation pieces, $\Omega_{r}$ is the rotational velocity of the permanent magnet rotor, and $\Omega_{s}$ is the rotational velocity of the flux modulator. $b_{r m}$ and $b_{\theta m}$ are the Fourier coefficients of the radial and circumferential flux density distribution, without the flux modulation pieces, respectively.

The Fourier coefficients for the radial and circumferential components of the flux density distribution resulting from the introduction of the flux modulation pieces are $\lambda_{r j}$ and $\lambda_{\theta j}$, respectively.

As stated above, the number of pole pairs in the space harmonics of flux density distribution produced by PMs is given by:

$$
\begin{aligned}
& p_{m, k}=\left|m p+k n_{s}\right|, \\
& m=1,3,5, \cdots, \infty, \\
& k=0, \pm 1, \pm 2, \pm 3, \cdots, \infty .
\end{aligned}
$$

The number of pole pairs of the low-speed rotor must be equal to $P_{m, k}$ for which $k \neq 0$ for torque transmission at a different speed. Furthermore, the rotational velocity of flux density space harmonics is given by:

$$
\Omega_{m, k}=\frac{m p}{m p+k n_{s}} \Omega_{r}+\frac{k n_{s}}{m p+k n_{s}} \Omega_{s} .
$$

If the ferromagnetic pole pairs are held stationary, $\Omega_{s}=$ 0 , the speed of the space harmonics of the low-speed rotor must be equal to $\Omega_{m, k}$.

Considering the combination of $m=1$ and $k=$ -1 , which results in the highest asynchronous space harmonic, the gear ratio of the high-speed to low-speed rotor can be given by:

$$
G_{r}=\frac{n_{s}-p}{p} .
$$

The gear ratio for the MG considered in this paper (with $p=4, n_{s}=27$ ) is $23 / 4=5.75$. Therefore, the conceptual design parameters of a single-sided axial flux magnetic gear are given in Table 1.
Table 1. Parameters of conceptual design.

\begin{tabular}{ll}
\hline Rated speed of high-speed rotor & $1500 \mathrm{rpm}$ \\
Number of high-speed PM rotor pole pairs & 4 \\
Number of low-speed PM rotor pole pairs & 23 \\
Number of ferromagnetic pole pieces & 27 \\
Outer radius & $100 \mathrm{~mm}$ \\
Axial length of each PM & $10 \mathrm{~mm}$ \\
Axial length of ferromagnetic pole pieces & $8 \mathrm{~mm}$ \\
Each air gap length & $2 \mathrm{~mm}$ \\
Active axial length of high-speed core & $15 \mathrm{~mm}$ \\
Active axial length of low-speed core & $5.5 \mathrm{~mm}$ \\
Remanence of PMs & $1.3 \mathrm{~T}$ \\
\hline
\end{tabular}

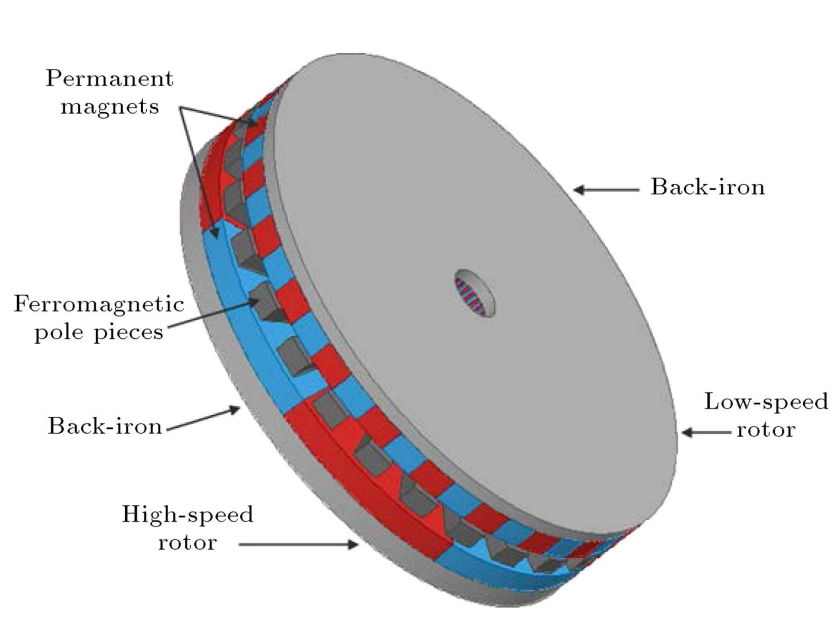

Figure 1. Single-sided axial flux magnetic gear.

\section{Single-sided axial flux MG}

Figure 1 shows an axial flux magnetic gear composed of 4-pole pair PMs in high-speed rotor, 23-pole pair PMs in low-speed rotor, and 27 ferromagnetic pole pieces between the high- and low-speed rotors. Permanent magnets are made of $\mathrm{Nd}-\mathrm{Fe}-\mathrm{B}$ material. The air gap length is supposed to be $2 \mathrm{~mm}$ between static and rotating parts.

Figure 2 shows the variations of the axial component of magnetic flux density, emanating from the highspeed permanent magnet rotor, and its corresponding harmonic spectrum in the air gaps adjacent to the lowspeed rotor.

It is seen that the presence of the steel pole pieces results in a number of asynchronous $(k \neq 0)$ space harmonics. The largest number is 23-pole pair space harmonic $(m=1, k=-1)$ interacting with the 23pole pair low-speed rotor PMs to transmit a torque at a rotational velocity of:

$$
\Omega_{l r}=\frac{p_{h}}{p_{h}-n_{s}} \Omega_{h r}=-\frac{4}{23} \Omega_{h r}
$$

where, $\Omega_{h r}$ and $\Omega_{l r}$ are the rotational velocities of the 


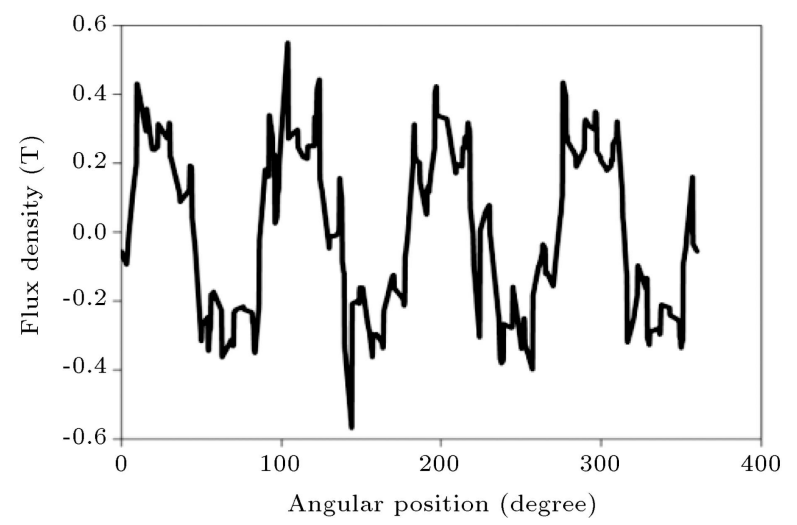

(a)

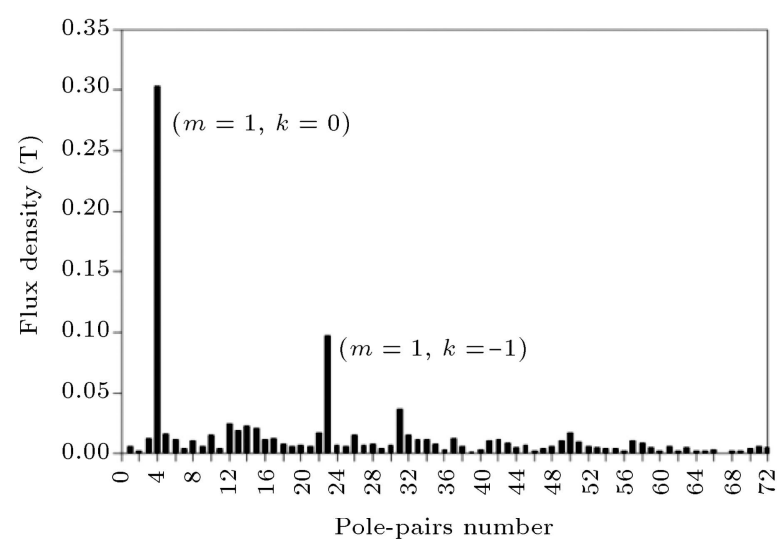

(b)

Figure 2. (a) Axial flux density wave form due to the high-speed PM rotor in the air gap adjacent to the low-speed rotor. (b) Space harmonic spectrum (radius $=$ $80 \mathrm{~mm})$.

high- and low-speed rotors, respectively. $p_{h}$ is the number of high-speed PM rotor pole pairs.

Likewise, the oscillations of the axial component of magnetic flux density, emanating from the low-speed PM rotor within the air gap adjacent to the high-speed rotor, are shown in Figure 3(a). It is clearly seen that there are 8 fluctuations, each of which corresponding to a pole.

The corresponding spectra of signal oscillations based on Fourier analysis are shown in Figure 3(b). It is seen that, subsequent to the flux density modulation, the dominant asynchronous space harmonics of the lowspeed PM rotor have 4-pole pairs $(m=1, k=-1)$ transmitting a torque at synchronous speed of:

$$
\Omega_{h r}=\frac{p_{l}}{p_{l}-n_{s}} \Omega_{l r}=-\frac{23}{4} \Omega_{l r},
$$

where, $p_{l}$ is the number of low-speed PM rotor pole pairs. It stands to reason that the speed of high speedrotor differs from that of the low-speed rotor.

The 3D FEM plots of the torque transmission capability of the individual PM rotors are shown in Figure 4.

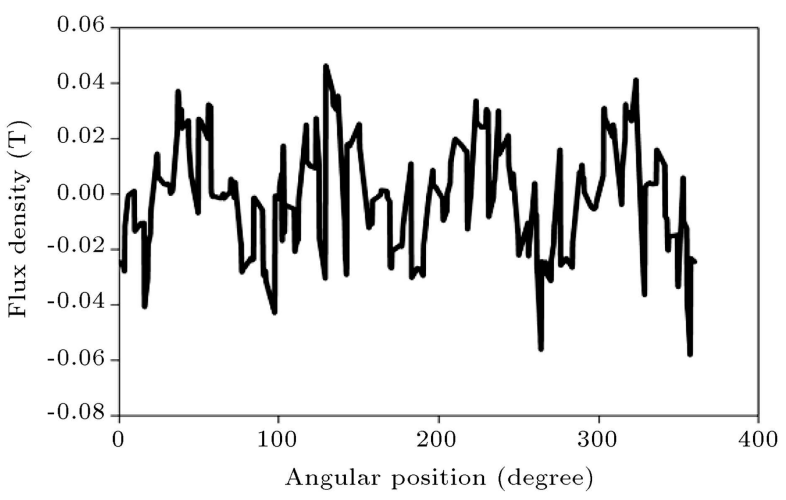

(a)

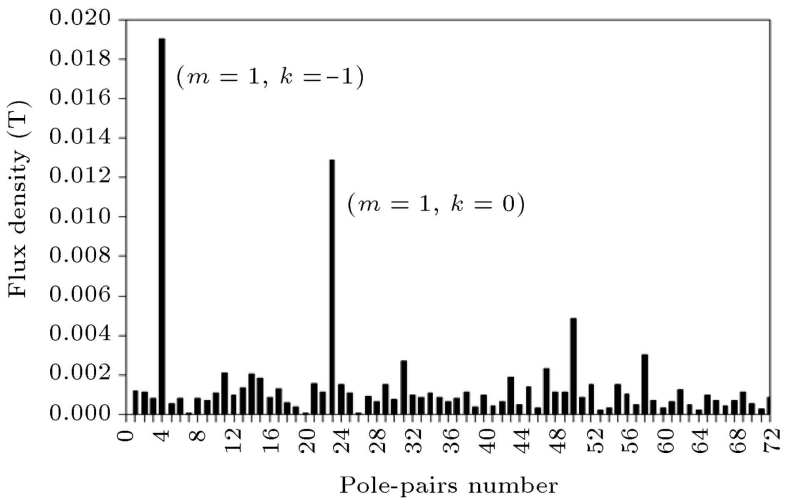

(b)

Figure 3. (a) Axial flux density wave form due to low-speed PM rotor within the air gap adjacent to the high-speed rotor. (b) Space harmonic spectrum (radius $=$ $80 \mathrm{~mm})$.

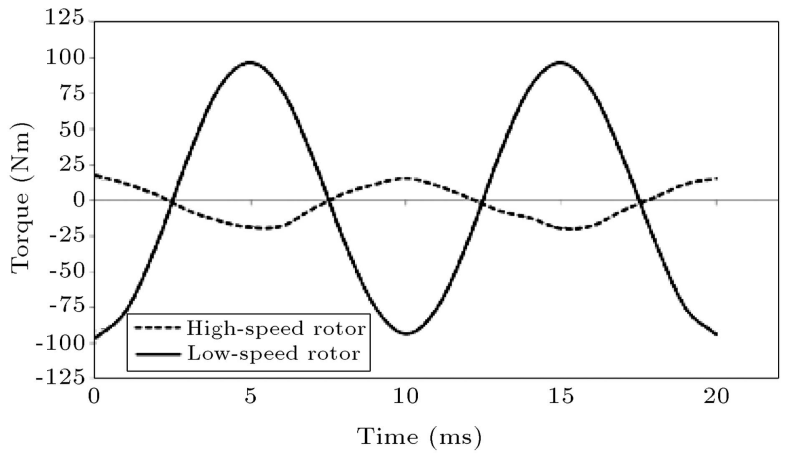

Figure 4. Plots of the torque transmission capability on high- and low-speed rotors of the single-sided axial flux MG.

The maximum output torque on the low-speed rotor and input torque on the high-speed (1500 rpm) rotor are close to $97 \mathrm{Nm}$ and $17 \mathrm{Nm}$, respectively.

\section{Proof-of-concept: Arcuate double-sided MG}

\subsection{Double-sided axial flux $M G$}

The preliminary study, presented in [13], has now been extended and improved with the new adopted analysis and an innovative double-sided axial flux MG. 


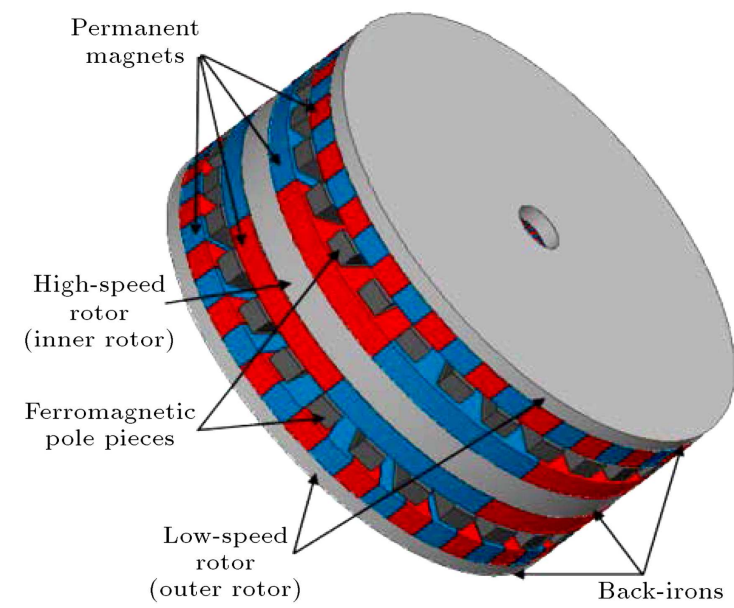

Figure 5. Schematic of the proposed double-sided axial-field MG.

Figure 5 provides a simplified model of the proposed double-sided axial flux MG that is easier to conceptualize. It comprises of two single-sided axial flux MGs, attached back to back from their highspeed (inner) cores. In the operation of this MG, the magnetic field components in the cumulative bundle give rise to an augmented torque profile, nearly twice of that of a single-sided MG. However, the high-speed core length of the double-sided MG increases to 30 $\mathrm{mm}$. There are two 8-pole pair high-speed Permanent Magnet (PM) rotors, two 23-pole pair external lowspeed PM rotors, and two sets of 23 ferromagnetic pole pieces.

Figure 6 shows the input and output torque variations on the high- and low-speed rotors in the double-sided axial flux MG, respectively. The highspeed rotor rotates at $1500 \mathrm{rpm}$, while the other rotor is kept stationary. It can be seen that the maximum input and output torque capacities are about $34 \mathrm{Nm}$ and $195 \mathrm{Nm}$, respectively.

\subsection{Arcuate double-sided $M G$}

The proposed double-sided MG yet falls short of field intensity and therefore torque enhancement. The

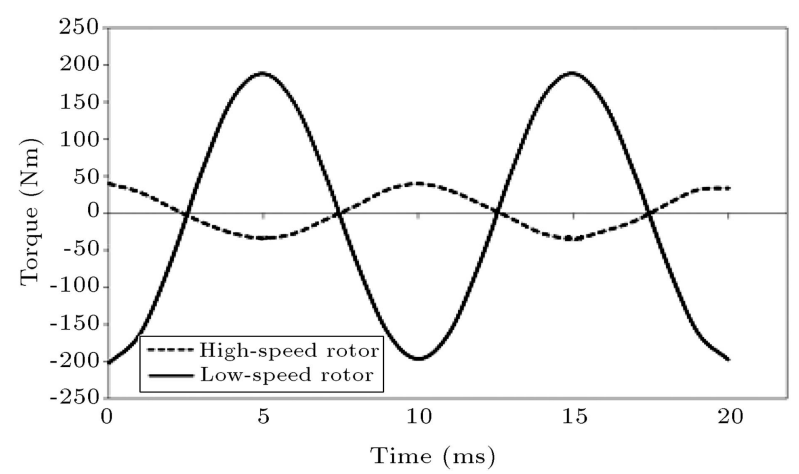

Figure 6. Plots of the torque transmission capability on high-speed and low-speed rotors for the proposed double-sided MG.

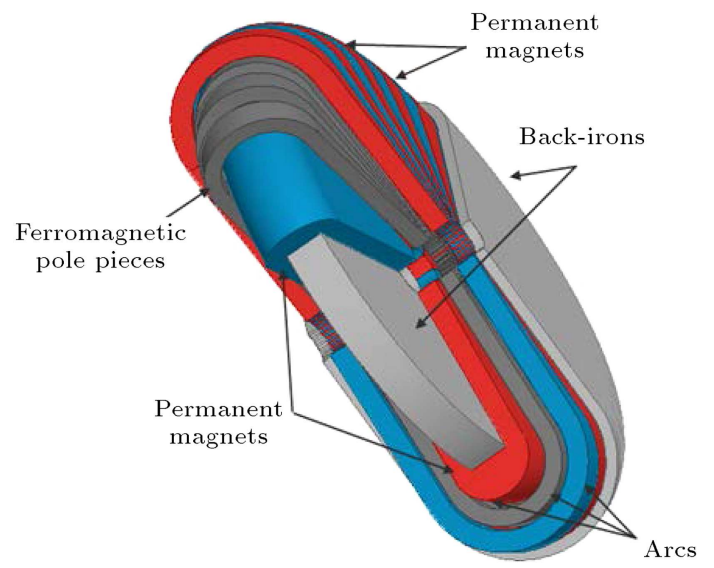

(a)

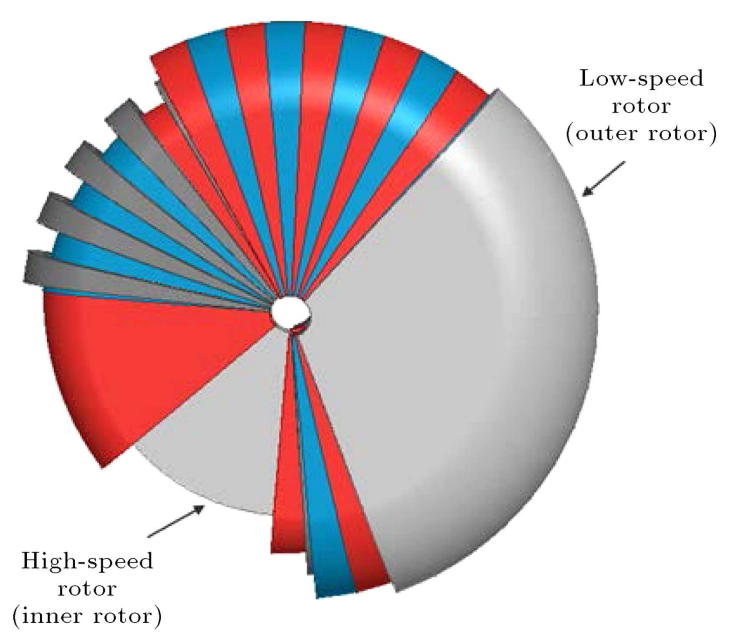

(b)

Figure 7. Plurality of magnetic ADSMG units: (a) Side view; and (b) front view.

present concept seeks to provide a precious guide and allows forecast of the ability to design and fabricate Arcuate Double-sided MG; henceforth, it is referred to as ADSMG for more effective approach to facilitate the field intensity and, thereby, further torque drive without changing the concept. In this and all the succeeding sections of the paper, only ADSMG will be considered.

Figure 7 depicts the ADSMG from different aspects. It collectively comprises a plurality of magnetic units which are spaced around the circumference of the rotatable member. Each magnetic unit extends along a direction substantially parallel to the central axis of the rotatable member and each magnetic unit is inversely aligned to its adjacent magnetic unit. The magnetic units are equidistantly spaced from one another, each of which containing a series of PMs been surface mounted on the high-speed (inner) and low-speed (outer) back iron cores. The number of PM pole-pairs of the highand low-speed rotors, and ferromagnetic pole pieces, together with their corresponding arcs are 4, 23, and 27 , respectively. 

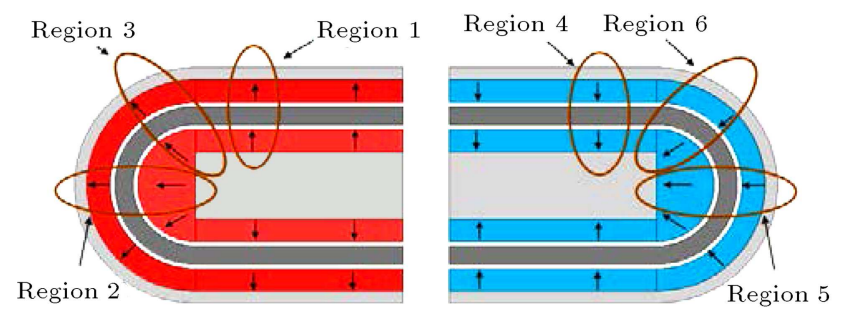

Figure 8. Simplified model of magnets magnetization in the ADSMG.

The arc units shown in Figure 7 benefit from radial fields composition so that they maximize magnetic flux density by bridging between $N$ and $S$ poles of the top side PMs and corresponding bottom side PMs. The PM arcs are radially magnetized and act as a radial MG. While, the arcs used for the high-speed PMs differ from those of the low-speed PMs. This makes the arc shaped magnetic fields encompassed in the entire spaces within ADSMG. Consequently, the amount of the output torque per volume is significantly improved in comparison with the conventional MGs.

Figure 8 shows a simplified model of the ADSMG with its magnets magnetized in $2 \mathrm{D} z-y$ plane directions that is easier to conceptualize. The specified regions were defined to serve as a frame of reference. The designated N-poles (left side) and S-poles (right side) show the arcuate and flat parts of the magnets magnetized in radial and axial directions, in which each individual perceives and interprets events.

\subsection{Simulation analysis for $A D S M G$}

This section explains the plots of the flux density distribution in the ADSMG obtained as a result of 3D magnetostatic and transient simulation process. This is performed to verify effectiveness of the theoretical equations used in the design process and validate the designed parameters. Automatic mesh generation and adaptivity for FEM plays an important role in electromagnetic systems. The optimal mesh density was generated, automatically, by the system according to the boundary curvature, thickness element number, strain and strain rate, and density window [14].

In this paper, the mesh generation based on adaptive meshing method, the generation/changing of a mesh, and finite element analysis was repetitively performed, and during these repetitions, the mesh was dynamically changed in accordance with the analysis results.

In the field calculation, the main flux and leakage flux paths were identified. The values of reluctance and permeance were assigned by which the main flux follows the effective magnetic link paths.

In order to increase the analysis accuracy, smaller mesh is preferably used at and around the ADSMG periphery. However, the conventional FEM is used to sense the localized flux density behaviors with underly-

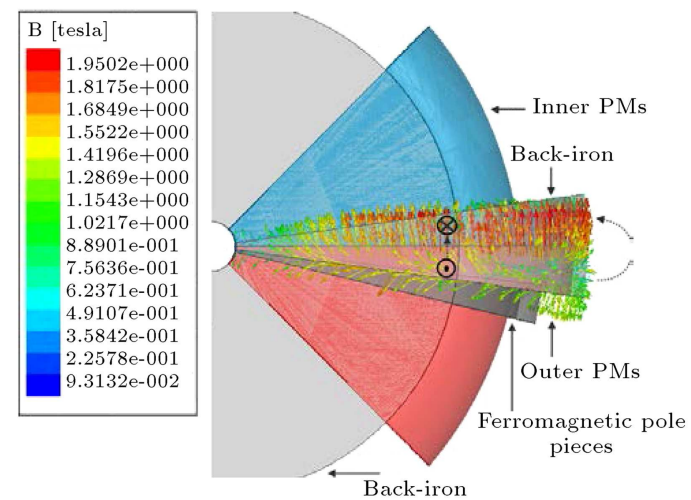

(a)

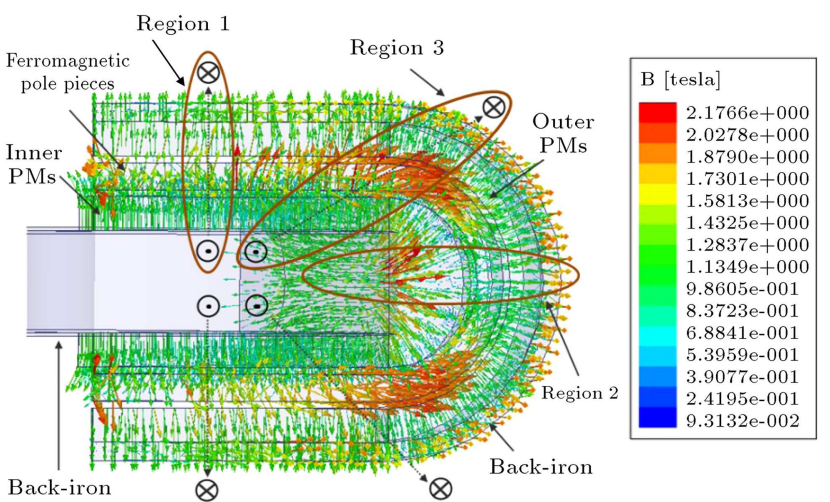

(b)

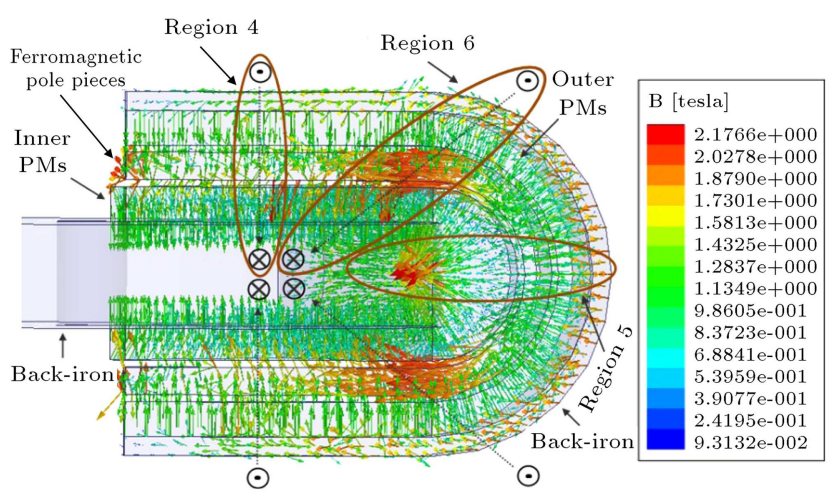

(c)

Figure 9. Vector plots of flux density distribution in ADSMG: (a) Front view; (b) lower cross-sectional view of (a); and (c) upper cross-sectional view of (a).

ing assumption that the regions have an appropriately chosen constant permeability.

The 3D direction and nature of the localized flux density behaviors in the most prominent regions, high- and low-speed PMs and back-irons are plotted and shown in the preferred layouts in Figure 7 and described by Figure 9(a) to (c). The symmetrical feature of this particular situation of rotating $\mathrm{MG}$ would cause the high- and low-speed poles experience similar flux patterns.

The direction and nature of the localized flux density, within the ADSMG PMs and back irons, are 
a good indication for the assessment of the ADSMG design and magnetic properties of the materials.

Generally, magnetic flux will take the easiest magnetic route from pole to pole. Based on this theory, Figure 9(a) clearly shows the distribution of the localized flux density vectors emanating from the outer rotor (low rpm) N-pole and detour via low-speed back iron core and entering their adjacent upper and lower S-poles.

The presence of the modulator exactly under the upper S-pole of the low-speed rotor provides a lower reluctance path for the flux density and results in higher field concentration in this region, as shown in Figure $9(\mathrm{a})$.

Figure 9(b) shows the distribution of flux density in a cross-section of the lower edge of the low-speed rotor's N-pole within Figure 9(a). It is seen that the localized flux densities emanate axially (region 1) and radially (region 2) from the high-speed core and emerge in low-speed back iron through the prescribed paths, air gap, and modulator under the combined effects of the merged flux densities. The reluctance in radial semicircle paths (region 2) is comparatively higher (wider cross-sectional area of the magnets) than that of axial paths (region 1) (see Figure 8). Thus, radial fields prefer to divert their paths and proceed via the modulator and join up with the axial fields in region 1 . As a result, the flux lines leaving the high-speed rotor's Npole prefer to proceed in the path in region 1 . As such, the flux densities are densely concentrated in region 3 and the proceeding flux densities within the modulator would face almost equal reluctance providing uniform distribution in radial and axial directions.

Figure $9(\mathrm{c})$ shows a plot of the flux distribution coming out from the low-speed S-pole to the ferromagnetic pole pieces, high-speed S-pole, and highspeed back iron in axial and radial directions. This corresponds to the cross-section of the upper edge of the low-speed S-pole shown in Figure 9(a). Similarly, in Figure 9(c), entrancing radial fluxes from lowspeed rotor S-pole to the modulator, due to the lower reluctance of region 4 with respect to region 5 , change their way to region 6 to expose in a path with lower reluctance. This causes increasing flux intensity in region 6 within the modulator.

The simultaneous interaction of the axial and radial fields with those of the opposite poles in neighboring ones in Figure 9(b) and (c) leads to increase in intensity of magnetic fields within the high-speed core. In addition, entrance of space vectors with different aspects and resultant vectors in high-speed core increases flux density in some regions of the highspeed core.

However, for the higher resolution and clarity purposes, the isovalue plot of the flux density distribution in some parts of the surfaces of ADSMG is shown in

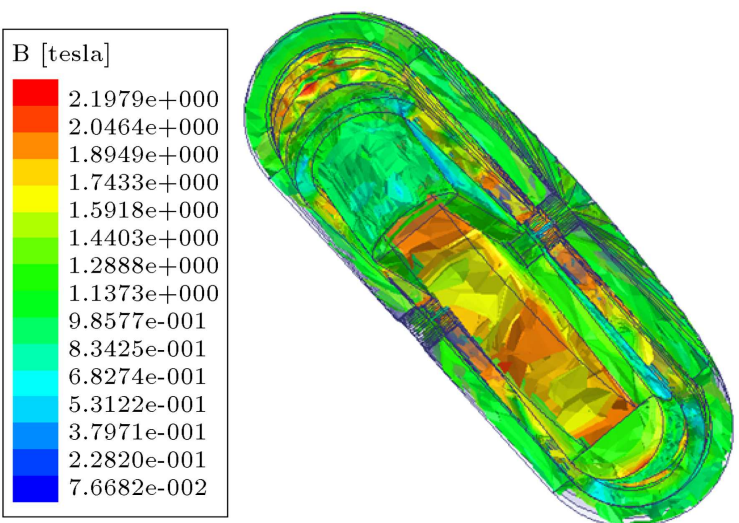

Figure 10. Plot of 3D magnetic flux density distribution in the ADSMG.

Figure 10 corresponding to complete Figure $7(\mathrm{a})$. It is observed that there is almost uniform flux density distribution in most parts of the ADSMG topology.

Moreover, careful inspection of Figure 10 shows that hot spots of around $2.20 T$ in the middle of semicircle of the modulator arise from non-uniformity of flux density. These localized high heat flux regions create hot spots that are significantly above the average saturating limit of the material. As the modulator geometry scales is comparatively smaller cross-sectional area, hot spots will become more pronounced.

As stated above, the field intensity within the ADSMG is directly proportional to its torque density. Thus, the field encompassment gives rise to more effective magnetic paths from three different crosssections and, thereby, a higher torque transmission is achieved.

The average magnetic torque developed on the high-speed and low-speed rotors can be obtained by calculating Maxwell stress in the inner and outer air gaps as:

$$
\begin{aligned}
& T_{m_{-} \text {in }}=\frac{L_{\mathrm{eff}} R_{\mathrm{in}}^{2}}{\mu_{0}} \int_{0}^{2 \pi} B_{r-\mathrm{in}} B_{\theta-\mathrm{in}} d \theta, \\
& T_{m_{\text {-out }}}=\frac{L_{\mathrm{eff}} R_{\mathrm{out}}^{2}}{\mu_{0}} \int_{0}^{2 \pi} B_{r-\text { out }} B_{\theta-\mathrm{out}} d \theta,
\end{aligned}
$$

where, $L_{\text {eff }}$ is active axial length, $R_{\text {in }}$ and $R_{\text {out }}$ are the radii of inner and outer air gaps, respectively, $B_{r-\text { in }}, B_{\theta-\text { in }}, B_{r-\text { out }}$, and $B_{\theta-\text { out }}$ are the radial and tangential flux densities in the inner and outer air gaps, respectively.

The correlation of the flux density and transmitted torque density of the ADSMG is given by Eqs. (12) and (13). The torque density is proportional to the integral of the radial and tangential flux densities in the inner and outer air gaps of $\mathrm{MG}$, respectively. 


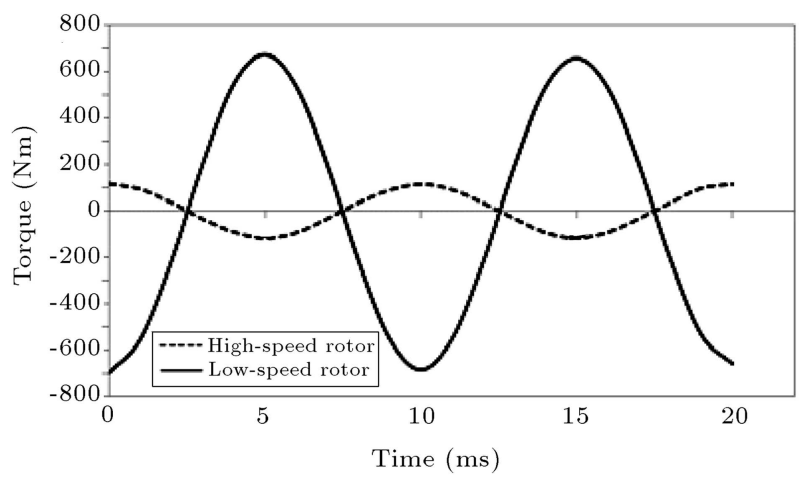

Figure 11. Torque transmission capability on high-speed and low-speed rotors of ADSMG.

Figure 11 shows the input and output torques' variations exerted on the high-speed (1500 rpm) and low-speed (stationary) PM rotors. It can be seen that the values of the input and output torques are about $116 \mathrm{Nm}$ and $667 \mathrm{Nm}$, respectively.

The torque variations start from initial position; the instant at which N-pole and S-pole of the highspeed rotor are aligned and overlapped, correspondingly, with those of the low-speed rotors and set to zero-angle along their $x$-axis.

However, the correlation between the torque peaks is very striking and reflects the likelihood that central field strength plays a key role in determining the best design geometries.

Table 2 shows the numerical values of the output torque volume and output torque density for singlesided axial flux, double-sided axial flux, and ADSMG. It is seen that ADSMG produces approximately $242 \%$ higher output torque (i.e., $87.8 \%$ output torque density) and $582.6 \%$ higher output torque (i.e., $88.4 \%$ output torque density) relative to the double-sided and single-sided MGs, respectively.

\subsection{Cogging torque study}

Cogging torque predominantly arises from the interaction between the magnetic field of high- and lowspeed rotors and angular variations of the modulator reluctance. This is due to a non-uniform air gap permeance (i.e., non-uniform flux distribution) resulted from the PMs constantly seeking a position of minimum reluctance. Cogging torque is the pulsating-speed that

Table 2. Comparison of different magnetic gears.

\begin{tabular}{lccc}
\hline $\begin{array}{c}\text { Magnetic } \\
\text { gear } \\
\text { type }\end{array}$ & $\begin{array}{c}\text { Output } \\
\text { torque } \\
(\mathbf{N m})\end{array}$ & $\begin{array}{c}\text { Volume } \\
\left.\mathbf{( m}^{\mathbf{3}}\right)\end{array}$ & $\begin{array}{c}\text { Output } \\
\text { torque } \\
\text { density } \\
\left(\mathbf{k N m} / \mathbf{m}^{\mathbf{3}}\right)\end{array}$ \\
\hline Single-sided MG & 97 & $1.649 * 10^{-3}$ & 58.8 \\
Double-sided MG & 195 & $3.299 * 10^{-3}$ & 59.0 \\
ADSMG & 667 & $6.019 * 10^{-3}$ & 110.8 \\
\hline
\end{tabular}

can blemish machined surfaces or reduce gear ratio accuracy and cause noise and vibration in the MG.

Generally, cogging torque can be calculated by the alteration of the total energy stored in the air gap with respect to the modulator position as [15-19]:

$$
T_{\text {cogging }}(\theta)=-\frac{1}{2} \varphi_{g}^{2} \frac{d \Re}{d \theta},
$$

where, $\varphi_{g}$ is the air gap flux, $\Re$ is the air gap reluctance, and $\theta$ is the relative angular modulator position.

It should be noted that by the higher PMs' field strength (increasing flux in the air gap), higher cogging torque occurs. The cogging torque increases linearly (non-uniform flux distribution in the air gap) while the modulator behaves linearly, but levels off and then decreases as the modulator begins to saturate. When the modulator material is saturated, the flux begins to distribute more evenly in the air gap, leading to cogging torque reduction.

Figures 12 and 13 show 3D FEM simulations of the cogging torques of the aforementioned three MGs for the low- and high-speed rotors, respectively. It can be seen that the average transmission torques of the high- and low-speed rotors are compatible with Table 2. The cogging torque values of the high-speed rotors in the single-sided, double-sided, and ADSMG topologies are $8.11 \mathrm{Nm}, 16.65 \mathrm{Nm}$, and $20.73 \mathrm{Nm}$, while for the low-speed rotors, they are $9.23 \mathrm{Nm}, 17.87 \mathrm{Nm}$, and $30.1 \mathrm{Nm}$, respectively.

However, the cogging torque simulations of the low-speed rotor show that the amplitude variations

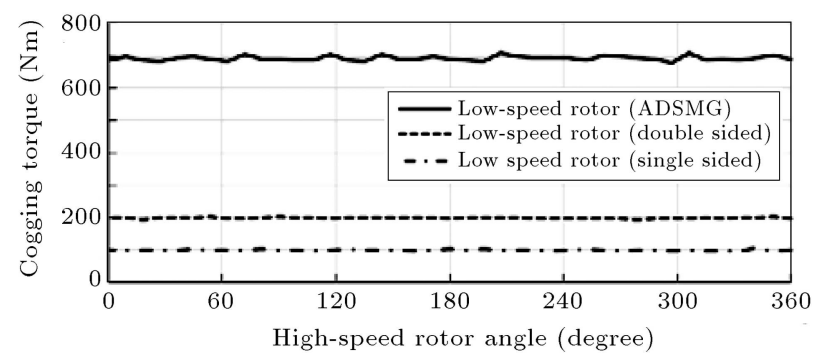

Figure 12. Computed cogging torque waveform of the low-speed rotor by 3-D FEM for single-sided, double-sided, and ADSMG topologies.

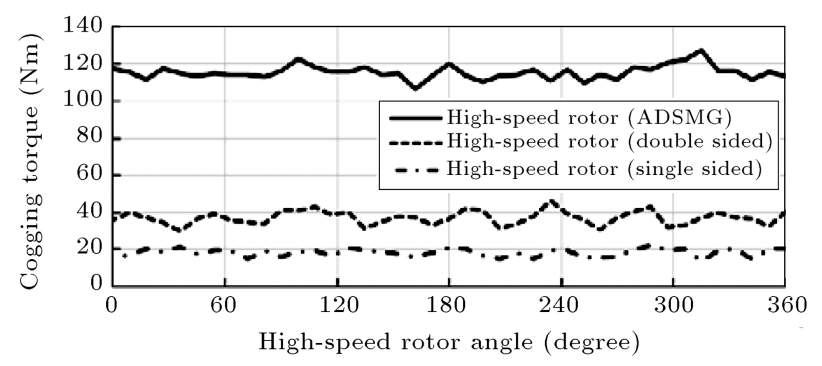

Figure 13. Computed cogging torque waveforms of the high-speed rotor by 3-D FEM for single-sided, double-sided, and ADSMG topologies. 


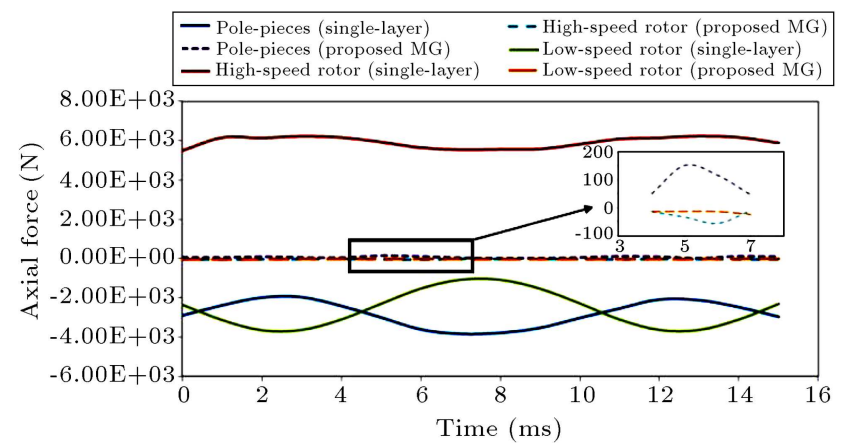

Figure 14. Axial forces on different parts of single-sided and ADSMG topologies.

percentage (Peak-Peak) in ADSMG is $4.51 \%$ while those of single-sided and double-sided are $9.5 \%$ and $9.1 \%$, respectively. This implies that the cogging torque in the proposed ADSMG has reduced by about $50 \%$ with respect to the other two MGs.

Contrary to the single-sided axial flux MG, the ADSMG topology is symmetrical; thus, the axial forces on its various parts are nearly balanced out and therefore negligible, as shown in Figure 14. The distinctive properties of ADSMG can prove its superiority in terms of disturbances in the air gap flux density, failures of bearings, vibration and noises, and finally, mechanical failures.

However, the axial force values on the single-sided axial flux MG are about 4 to $6 \mathrm{kN}$. While, those of the ADSMG rotors are around 50 to $150 \mathrm{~N}$.

\section{Conclusion}

The principle of operation and the torque transmission capability of the DSMG topology were analytically studied and validated by 3D FEM analysis.

It was shown that field intensity arrangement plays an important role in the economics of $\mathrm{MG}$ designs. The importance of 'shaping' the leakage magnetic field in the end region of the PMs (arcuate PMs) was demonstrated, as it increased the influence of the effective field components acting on the torque enhancements by $87.8 \%$ and $88.4 \%$ compared to its corresponding double- and single-sided axial flux MGs, respectively.

The simulation results also indicated that the cogging torque in the proposed approach was reduced by about $50 \%$ as compared with its single- and doublesided axial flux MG. Although cogging torque cannot be completely eliminated, further reduction of the cogging torque in ADSMG is a challenging task for the authors' future research, using magnet and modulator shaping, sizing, and skewing.

Furthermore, the correlation of the flux distribution and the corresponding torque density results established the validity of the models. Thus, the simulation results can be used to accurately predict the behavior of an actual ADSMG.

The information can be thought of as a performance signature of the ADSMG. From this data, many important parameters can be extracted to verify whether the design calculations, especially finite element analysis results, are in good correlation with a prototype.

However, the present approach has been described with reference to an exemplary ADSMG. It will supply a precious guide and allows forecast of the ability to design and fabricate an ADSMG for industrial equipment. As such, feasible study for implementing a laboratory model ADSMG is being carried out in High Voltage and Magnetic Material Research Center at IUST by considering undesirable effects of:

a) Hot spots in the modulator magnetic material,

b) Harmonics of the magnetic flux within the air gaps on the gear ratio.

\section{References}

1. Chau, K.T., Zhang, D., Jiang, J.Z., Liu, C. and Zhang, Y. "Design of magnetic-geared outer-rotor permanentmagnet brushless motor for electric vehicles", IEEE Trans. on Magn., 43, pp. 2504-2506 (2007).

2. Jian, L., Chau, K.T. and Jiang, J.Z. "A magneticgeared outer-rotor permanent-magnet brushless machine for wind power generation", IEEE Trans. on Ind. Appl., 45, pp. 954-962 (2009).

3. Ho, S.L., Niu, S. and Fu, W.N. "Design and analysis of a novel axial-flux electric machine", IEEE Trans. on Magn., 47, pp. 4368-4371 (2011).

4. Hesmondhalgh, D.E. and Tipping, D. "A multielement magnetic gear", IEEE Trans. on Ind. Appl., 45, pp. 954-962 (2009).

5. Tsurumoto, K. and Kikushi, S. "A new magnetic gear using permanent magnet", IEEE Trans. on Magn., MAG-23, pp. 3622-3624 (1987).

6. Atallah, K. and Howe, D. "A novel high-performance magnetic gear", IEEE Trans. on Magn., 37, pp. 28442846 (2001).

7. Mezani, S., Atallah, K. and Howe, D. "A highperformance axial-field magnetic gear", AIP Journal of Applied Physic, 99, pp. 08R303-08R303-3 (2006).

8. Jian, L., Xu, G. and Song, J. "Optimum design for improving modulating-effect of coaxial magnetic gear using response surface methodology and genetic algorithm", Progress in Electromagnetic Research, 116, pp. 297-312 (2011).

9. Fukuoka, M., Nakamura, K. and Ichinokura, O. "A method for optimizing the design of SPM type magnetic gear based on reluctance network analysis", Proc. 20th Int. Conf. on Electrical Machines (ICEM), pp. 30-35 (2012). 
10. Jian, L., Chau, K.T., Li, W. and Li, J. "A novel coaxial magnetic gear using bulk HTS for industrial applications", IEEE Trans. on Applied Superconductivity, 20, pp. 981-984 (2010).

11. Jian, L. and Chau, K.T. "A coaxial magnetic gear with halbach permanent-magnet arrays", IEEE Trans. on Energy Conv., 25(2), pp. 319-328 (2010).

12. Lubin, T., Mezani, S. and Rezzoug, A. "Analytical computation of the magnetic field distribution in a magnetic gear", IEEE Trans. on Magn., 46(7), pp. 2611-2621 (2010).

13. Atallah, K., Calverley, S.D. and Howe, D. "Design, analysis and realization of a high-performance magnetic gear", IEE Proc. Electric Power Appl., 151, pp. 135-143 (2004).

14. Faghihi, F. and H. Heydari, H. "Reduction of leakage magnetic field in electromagnetic systems based on active shielding concept verified by eigenvalue analysis", Progress in Electromagnetics Research, PIER, 96, pp. 217-236 (2009).

15. Bretón, C., Bartolomé, J., Benito, J.A., Tassinario, G., Flotats, I., Lu, C.W. and Chalmers, B.J. "Influence of machine symmetry on reduction of cogging torque in permanent magnet brushless motors", IEEE Trans. on Magn., 36, pp. 3819-3823 (2000).

16. Hwang, S.M., Eom, J.-B., Jung, Y.-H., Lee, D.W. and Kang, B.-S. "Various design techniques to reduce cogging torque by controlling energy variation in permanent magnet motors", IEEE Trans. on Magn., 37, pp. 2806-2809 (2001).

17. Li, T. and Slemon, G. "Reduction of cogging torque in permanent magnet motors", IEEE Trans. on Magn., 24, pp. 2901-2903 (1988).

18. Niguchi, N. and Hirata, K. "Cogging torque analysis of magnetic gear", IEEE Trans. on Industrial Electronics, 59, pp. 2189-2197 (2012).

19. Dosiek, L. and Pillay, P. "Cogging torque reduction in permanent magnet machines", IEEE Trans. on Industry Applications, 43, pp. 1565-1571 (2007).

\section{Biographies}

Seyed Ahmadreza Afsari received the BS degree from K. N. Toosi University of Technology, Tehran, Iran, in 2007 and the MSc degree from the University of Kashan, Kashan, Iran, in 2010, both in Electrical Power Engineering. He is currently working toward the $\mathrm{PhD}$ degree in Electrical Engineering at the High Voltage and Magnetic Material Research Center, Department of Electrical Engineering, Iran University of Science and Technology, Tehran, Iran. His research interests are magnetic gears, analytical methods, finite-element-method-based simulation techniques, optimization methods, and applied superconductors in power systems.

Hossein Heydari received the BS degree in Electrical Engineering and the MSc degree in Power Electronics from Loughborough University, Loughborough, U.K., in 1985 and 1987, respectively, and the $\mathrm{PhD}$ degree in Transformer Core Losses from the University of Wales, Cardiff, U.K., in 1993. Following graduation, he joined Iran University of Science and Technology (IUST), Tehran, Iran, as an Academic Member (Lecturer) of the Electrical Power Group, and was also appointed as the Director of the High Voltage and Magnetic Materials Research Center. He is currently with the Center of Excellence for Power System Automation and Operation, IUST. His research interests are EMC considerations in power systems, magnetic gears, fault current limiters, and applied superconductivity in power systems.

Erfan Bashar received the BS degree from Islamic Azad University, South Tehran Branch, Tehran, Iran, in 2009 and the MSc degree from Iran University of Science and Technology (IUST), Tehran, Iran, in 2012, both in Electrical Power Engineering. He is currently working with the High Voltage and Magnetic Materials Research Center at IUST. His research interests are magnetic gears, electrical machines, optimization methods, and power system studies. 